\title{
Efficacy and safety of percutaneous transforaminal endoscopic discectomy in the treatment of lumbar spinal stenosis combined with osteoporosis
}

\author{
iD Xiaobin $\mathrm{Gu}^{\mathbf{1}}$ \\ iD Wenhui Zhu' \\ (iD) Haiyi He ${ }^{1}$ \\ (iD) Zili Wang ${ }^{1}$ \\ Shaolong Ding ${ }^{1}$ \\ iD Gaosheng Guo ${ }^{1}$
}

1. Department of Orthopedics, Sanmenxia Central Hospital, Sanmenxia 472000, China

http://dx.doi.org/10.1590/1806-9282.65.6.779

\section{SUMMARY}

OBJECTIVE: To investigate the efficacy and safety of percutaneous transforaminal endoscopic discectomy (PTED) in the treatment of lumbar spinal stenosis (LSS) combined with osteoporosis.

METHODS: Eighty patients with LSS combined with osteoporosis were divided into a control and PTED group, which received conventional transforaminal lumbar interbody fusion and PTED, respectively. The surgical indications, incision visual analogue scale (VAS), lumbar and leg pain VAS, lumbar Japanese Orthopaedic Association (JOA) and Oswestry disability index (ODI) scores, bone mineral density (BMD), and adverse reactions were observed.

RESULTS: Compared with the control group, in the PTED group, the operation time, bleeding loss and hospitalization duration, incision VAS scores at postoperative 12, 24 and $48 \mathrm{~h}$ and lumbar and leg pain VAS and lumbar ODI scores on postoperative 6 months were significantly decreased $(P<0.01)$, and the lumbar JOA score on postoperative 6 months was significantly increased $(P<0.05)$. There was no significant difference in BMD between two groups $(P>0.05)$. Compared with the control group, in the PTED group, the total effective rate was significantly higher $(P<0.05)$, and the incidence of adverse reactions was significantly lower $(P<0.05)$.

CONCLUSIONS: PTED is safe and effective in the treatment of LSS combined with osteoporosis.

KEYWORDS: Diskectomy, Percutaneous. Spinal stenosis. Osteoporosis. Efficacy. Safety.

\section{INTRODUCTION}

Lumbar spinal stenosis (LSS) is a common disease in the elderly. It refers to a series of syndromes caused by cauda equina or nerve root compression due to the spinal stenosis. The main pathogeny of LSS is lumbar disc herniation, osteophyte formation, facet hyperplasia, and yellow ligament hyper- trophy'. Osteoporosis is a systemic skeletal disease caused by the reduction of bone mass in the body. It is characterized by bone microstructure degradation and increased bone fragility. Vertebral compression fractures can occur in severe osteoporosis patients ${ }^{2}$. The elderly LSS patients often have oste- 
oporosis as a complicating factor. The main manifestations in these patients are lumbar and leg pain, severe intermittent claudication, and even complete bedridden ${ }^{3}$. LSS combined with osteoporosis causes great damage to the body. Patients with LSS combined with osteoporosis are susceptible to a variety of internal medical diseases, such as coronary heart disease, hypertension, diabetes, and so on, of which the treatment is difficult ${ }^{4}$. Percutaneous transforaminal endoscopic discectomy (PTED) is a surgical way to reduce the edema and aseptic inflammation of the nerve root. It has the advantages of being widely indicated, having a small trauma surface, low influence on body appearance, rapid recovery, simple postoperative care, and low cost ${ }^{5-}$ 7. In addition, PTED does not affect remedial treatment after surgery failure ${ }^{8}$. This study investigated the efficacy and safety of PTED in the treatment of LSS combined with osteoporosis. The objective was to provide a reference for further clinical application of this treatment strategy.

\section{METHODS}

\section{Subjects}

A total of 80 patients with LSS combined with osteoporosis and treated in Sanmenxia Central Hospital (Sanmenxia, China) from March 2015 to June 2017 were enrolled in this study. There were 50 males and 30 females. The age of patients was 56-83 years, with an average of $64.31 \pm 6.22$ years. The disease course ranged from 1 month to 11 years. The patients were randomly divided into control and PTED group, with 40 cases in each group. This study was approved by the ethics committee of Sanmenxia Central Hospital. Written informed consent was obtained from all participants.

\section{Inclusion and exclusion criteria}

The inclusion criteria were as follows: i) the patients had symptoms of intermittent claudication, lumbar and leg pain, numbness and feeling cold; ii) the signs included lumbar scoliosis, paravertebral radiation-induced pain and lower extremity abnormal sensation, muscle strength, and tendon reflex; iii) the $\mathrm{T}$ value of bone mineral density (BMD) was lower than the average value of healthy individuals, with 2.5 standard deviation; iv) the CT and MRI confirmed the lumbar degenerative change and lateral recess stenosis. The exclusion criteria were as follows: i) significant organ dysfunction, bleeding tendency, and neuronal diseases; ii) pregnancy; iii) mental disorders; iv) central spinal canal stenosis; v) lumbar spondylolisthesis with $>$ II degree.

\section{Surgical methods}

The control group received the conventional transforaminal lumbar interbody fusion treatment. The patients were placed in the prone position and received general anesthesia. The lesion was located by C-arm X-ray. The vertebral plate was separated to fully expose the lesion, followed by re-positioning to determine the direction and length of the incision. Screw implantation and decompression treatment were conducted. The bilateral articular processes were retained. The fusion device was installed. After washing the wound and disinfecting it, the incision was closed. The PTED group received PTED treatment. The patients were in the lateral recumbent position and received local anesthesia. The skin location for the needle was determined by C-arm X-ray at orthophoric and lateral position, and the puncture point was determined under orthophoric X-ray. The pain-evoked test and discography were performed to verify the diagnosis. The intervertebral foramen was enlarged, and the working channel was placed. The percutaneous transforaminal endoscope was placed through the working channel. The working channel and the endoscope direction were adjusted to probe the spinal canal. The protruding blue nucleus pulposus was removed using nucleus pulposus rongeur. The nerve root $270^{\circ}$ complete decompression and good self-pulsation indicated the completion of removal. The radiofrequency ablation was conducted to clear the residual nucleus pulposus in the intervertebral disc, followed by hemostasis. One milliliter compound beclomethasone was injected into the wound. The outer tube was removed, followed by the sterile dressing of the small incision.

\section{Postoperative treatment}

In the course of treatment, all patients received antibiotic prophylaxis treatment, followed by dewatering and hemostasis. After 1-3 days from treatment, the patients performed ambulation. The auxiliary leg-raising exercise was conducted during the break to prevent nerve root adhesion. The patients were required to wear the belt in the ambulation. Waist movement was avoided for 6 weeks after the 
treatment. Moderate back muscle training was performed. Patients received routine anti-osteoporosis treatment by taking a calcium preparation or active vitamin D3, or intramuscular injection of salmon calcitonin.

\section{Evaluation of surgical indicators and treatment-related scores}

The surgical indicators, including operative time, blood loss, and hospitalization duration in both groups were recorded. At $12 \mathrm{~h}, 24 \mathrm{~h}$, and $48 \mathrm{~h}$ after the operation, the incision pain felt by the patients was evaluated using the visual analog scale (VAS). One day before the operation and six months after it, the lumbar and leg pain of patients were evaluated using the VAS. The VAS scores were as follows ${ }^{9}$ : 0 points: no pain; $1-3$ points: mild pain; patient could bear it; 4-6 points: obvious pain; sleep was affected; patient could bear it; 7-10 points: gradually intense pain; patients could not bear it. The improvement of lumbar function was evaluated using the Oswestry Disability Index $(\mathrm{ODI})^{10}$ and the Japanese Orthopaedic Association $(\mathrm{JOA})^{11}$ scores. The ODI contained 10 questions about daily activities, including inventories of pain intensity, personal care, lifting, walking, sitting, standing, sleeping, sexual life, social life, and traveling. Each question was rated on a scale from 0 to 5 points. The ODI scores ranged from 0 to 50 . The higher scores indicated greater disability. The JOA scores included 9 points in subjective symptoms, 6 points in clinical signs, 14 points in daily activity, and 6 points in bladder function. The lower score indicated severer dysfunction.

\section{Evaluation of treatment efficacy}

There are four grades for clinical cure, improvement, and ineffectiveness. i) cured: the clinical symptoms and signs disappeared, the functional activities were normal, the straight leg elevation was by more than $70^{\circ}$; ii): markedly effective: the clinical symptoms and signs basically disappeared, the functional activities were normal, the straight leg elevation was by more than $45^{\circ}$; iii) effective: the clinical symptoms and signs were mitigated, the straight leg elevation was by more than $30^{\circ}$; iv) ineffective: the clinical symptoms and signs did not obviously change. The effective rate (\%) was calculated as follows: (cured number + markedly effective number + effective number) / total number] $\times 100$.

\section{Examination of BMD}

One day before the operation and six months after it, the BMD values of lumbar vertebrae (L1-4), femoral neck, Ward's triangle, and greater trochanter were measured using KJ7000 X-ray bone densitometer (Nanjing Kejin Industrial Co., Ltd., Nanjing, China).

\section{Statistical analysis}

All statistical analyses were carried out using SPSS22.0 software (SPSS Inc., Chicago, IL, USA). The enumeration data were presented as number and rate and were compared using the $\chi^{2}$ test. The measurement data were presented as mean \pm standard deviation and were compared using the t-test. $\mathrm{P}<0.05$ was considered statistically significant.

\section{RESULTS}

\section{General data of subjects}

In the control group, there were 22 males and 18 females; the age of patients was $64.43 \pm 3.58$ years, and the disease course was $6.63 \pm 2.15$ years. In the PTED group, there were 20 males and 20 females; the age of patients was $65.02 \pm 4.13$ years, and the disease course was $6.76 \pm 2.21$ years. There was no significant difference in age, gender, or disease course between both groups $(\mathrm{P}>0.05)$.

\section{Comparison of operation time, blood loss and} hospitalization duration between both groups

The operation time, blood loss and hospitalization duration in the control group were $93.51 \pm 15.16 \mathrm{~min}$, $175.84 \pm 26.7 \mathrm{ml}$ and $8.27 \pm 2.54$ days, respectively, and those in PTED group were $58.27 \pm 9.07 \mathrm{~min}, 14.03 \pm 4.07$ $\mathrm{ml}$ and $4.42 \pm 1.05$ days, respectively. Compared with the control group, the operation time, blood loss, and hospitalization duration in the PTED group were significantly decreased $(\mathrm{P}<0.05)$.

\section{Comparison of incision VAS score between both groups}

At $12 \mathrm{~h}, 24 \mathrm{~h}, 48 \mathrm{~h}$, and $72 \mathrm{~h}$ after the surgery, the incision VAS scores in the control group were $8.72 \pm 2.01,7.31 \pm 1.19,6.02 \pm 0.97$ and $2.88 \pm 1.04$ points, respectively, and those in the PTED group were $3.61 \pm 1.35,2.24 \pm 1.11,1.21 \pm 0.49$ and $0.69 \pm 0.16$ points, respectively. The incision VAS score at each time in the PTED group was significantly lower than in the control group $(\mathrm{P}<0.05)$. 
FIGURE 1. COMPARISON OF LUMBAR JOA AND ODI BETWEEN THE TWO GROUPS.

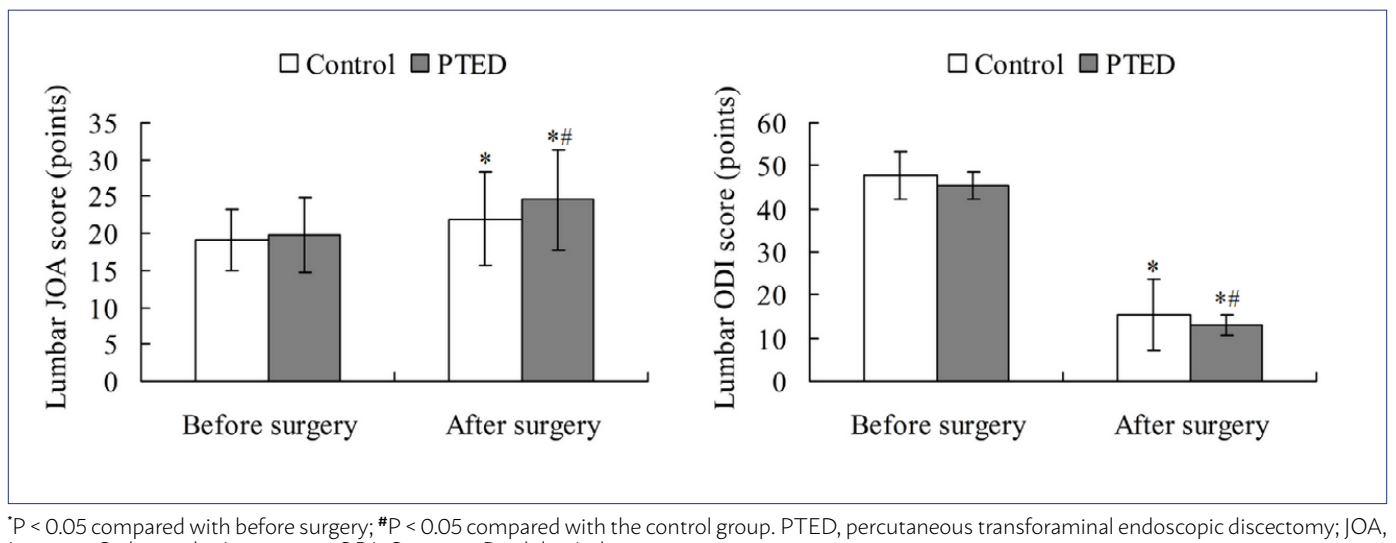
Japanese Orthopaedic Association; ODI, Oswestry Disability Index.

FIGURE 2. COMPARISON OF LUMBAR VERTEBRAE (L1-4) AND FEMORAL NECK BMD BETWEEN THE TWO GROUPS (G/CM²).

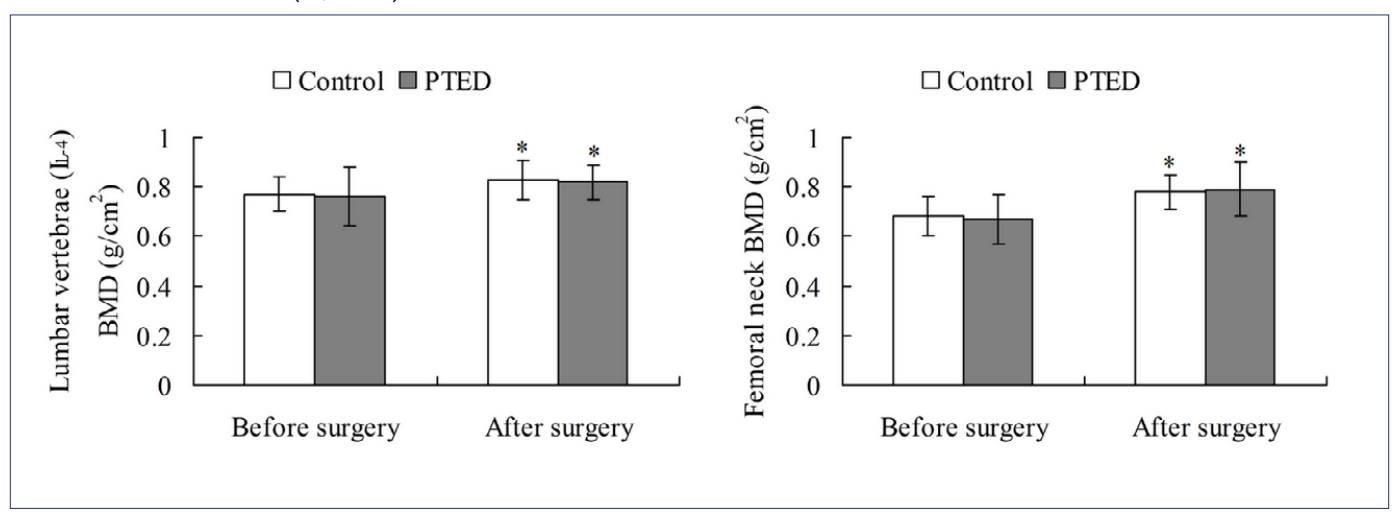

$P<0.05$ compared with before surgery. PTED, percutaneous transforaminal endoscopic discectomy; BMD, bone mineral density.

FIGURE 3. COMPARISON OF WARD'S TRIANGLE AND GREATER TROCHANTER BMD BETWEEN THE TWO GROUPS (G/CM²).

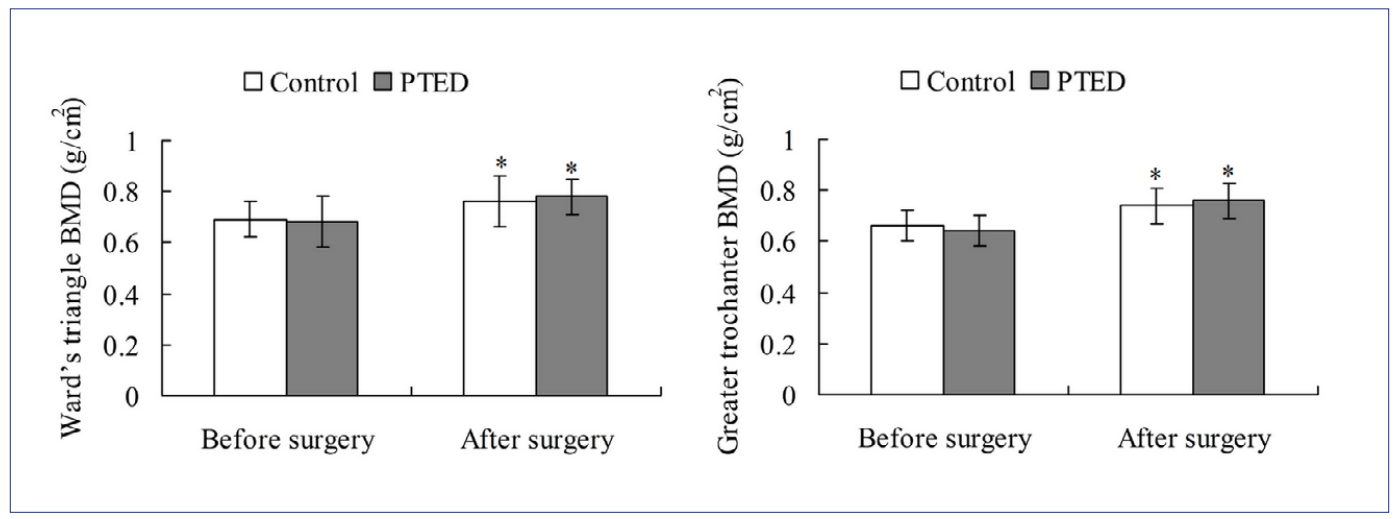

$P<0.05$ compared with before surgery. PTED, percutaneous transforaminal endoscopic discectomy; BMD, bone mineral density.

\section{Comparison of lumbar and leg pain VAS} scores between both groups

Before surgery, the lumbar VAS scores in the control and PTED groups were $5.89 \pm 1.57$ and $6.14 \pm 1.93$ points, respectively, with no significant difference between them $(\mathrm{P}>0.05)$. After surgery, the lumbar VAS scores in the control and PTED groups were $2.89 \pm 1.86$ and $1.70 \pm 0.81$ points, respectively, which were significantly lower than before surgery $(\mathrm{P}<0.05)$. Before surgery, the leg pain VAS scores in the control and PTED groups were $7.79 \pm 1.77$ and $7.54 \pm 1.87$ points, respectively, with no significant difference between them $(\mathrm{P}>0.05)$. After surgery, the leg pain VAS scores in control and PTED groups were 
$1.77 \pm 0.45$ and $1.42 \pm 0.40$ points, respectively, which were also significantly lower than before surgery $(\mathrm{P}<0.05)$. In addition, the lumbar and leg pain VAS scores after surgery in the PTED group were significantly lower than those in the control group, respectively $(\mathrm{P}<0.05)$.

\section{Comparison of lumbar JOA and ODI scores}

\section{between both groups}

Compared with before the surgery, after six postoperative months, the lumbar JOA score in each group was significantly increased $(\mathrm{P}<0.05)$, and the lumbar ODI score in each group was significantly decreased $(\mathrm{P}<0.05)$. In addition, after six postoperative months the lumbar JOA score in the PTED group was significantly higher than that in the control group $(\mathrm{P}$ $<0.05$ ), and the lumbar ODI score in the PTED group was significantly lower than that in the control group $(\mathrm{P}<0.05)$ (Figure 1).

\section{Comparison of total treatment efficacy between both groups}

There were $6,10,13$ and 11 cases with cured, remarkably effective, effective, and ineffective treatment outcome in the control group, respectively, and $13,15,8$, and 4 cases with cured, remarkably effective, effective, and ineffective outcome in the PTED group, respectively. The effectiveness rate in the PTED group was $90.00 \%$ (36/40), which was significantly higher than the $72.50 \%(29 / 40)$ in the control group $(\mathrm{P}<0.05)$.

\section{Comparison of BMD between both groups}

After six months of treatment, the BMD values of lumbar vertebrae (L1-4), femoral neck, Ward's triangle and greater trochanter in each group were significantly increased, compared with before treatment $(\mathrm{P}<0.05)$. Before and after treatment, there was no significant difference in each index between the two groups $(\mathrm{P}>0.05$ ) (Figure 2; Figure 3).

\section{Adverse reactions and follow-up}

During the surgery, there was 1 case of transient nerve stimulation reaction in the PTED group, which was recovered in 2 weeks after the operation. In the control group, there were 3 cases of nerve injury, 2 cases of dural bursa rupture, and 1 case of infection, which were improved after active treatment. The incidence of adverse reaction in the PTED group was $2.5 \%(1 / 40)$, which was significantly lower than $15 \%$
(6/40) in the control group $(\mathrm{P}<0.05)$. The follow up on the 12th month after the operation showed that the patients in both groups were all healed.

\section{DISCUSSION}

PTED is similar to spinal endoscopy. In PTED, the specially designed intervertebral foraminoscope and correspondingly equipped minimally invasive spinal surgical instrument, an imaging device, bipolar electrocoagulation, and ozone therapy apparatuses together make up the minimally invasive spinal surgery system ${ }^{12}$. The surgery is performed under a sober state with local anesthesia. The skin incision is small, with no interference to the spinal canal. The trauma is little, with no destruction of paravertebral muscles or ligaments, or influence on the stability of the spine. When completely removing the protruding or procident nucleus pulposus, the bone hyperplasia can also be removed, and the spinal canal stenosis is cleared $^{13}$. In addition, the damaged fibrous annulus can be repaired using radiofrequency ablation technology ${ }^{14}$. As PTED is performed outside the fibrous ring, the integrity of the fibrous ring and stability of the spine can be maintained to the maximum extent ${ }^{12}$. Among the same kind of surgeries, PTED causes the least trauma to patients and has the best treatment efficacy. It has been called the ultimate therapy method for the treatment of LSS.

In the present study, PTED was performed to treat LSS combined with osteoporosis. The efficacy and safety of PTED were compared with the conventional transforaminal lumbar interbody fusion treatment. Results showed that, compared with the control group, in the PTED group, the surgical indicators were clearly improved. In addition, after six months of the surgery, the lumbar and leg pain VAS scores and lumbar ODI score in the PTED group were significantly lower than in the control group $(\mathrm{P}<0.01)$, and the lumbar JOA score in the PTED group was significantly higher than that in control group $(\mathrm{P}<$ 0.01). The effectiveness rate in the PTED group was significantly higher than in the control group $\mathrm{B}(\mathrm{P}<$ 0.05), and the incidence of adverse reactions in the PTED group was significantly lower than in the control group $(\mathrm{P}<0.05)$. These results were basically in accordance with the results of previous studies ${ }^{15,16}$, which indicate that PTED is effective and safe in the treatment of LSS combined with osteoporosis. In addition, after six months, there was no significant dif- 
ference in BMD between the two groups $(\mathrm{P}>0.05)$. This indicates that PTED has no obvious effect on the BMD for patients with LSS combined with osteoporosis who received routine anti-osteoporosis treatment.

In the recovery period of patients with LSS combined with osteoporosis, the spleen, stomach, and kidney functions are weak, so more attention should be paid to the nutrition intake and calcium supplement ${ }^{17}$. The nursing staffs should advise the patients to eat more calcium-containing foods, such as milk, eggs, lean meat and so on, and the patients should receive routine anti-osteoporosis treatment by taking calcium preparation or active vitamin D3, or intramuscular injection of salmon calcitonin. In addition, during the treatment, the patients should increase back, waist, and limb muscle exercises.

In conclusion, compared with the conventional treatment, PTED has more advantages for surgical indications and improved treatment efficacy, with less adverse reactions. It is an effective and safe method in the treatment of LSS combined with osteoporosis. This study still has some limitations. Firstly, the sample size is relatively small. In future studies, the sample size should be further increased to obtain more satisfactory outcomes. Secondly, we did not investigate whether there are correlations among different indicators in LSS combined with osteoporosis. This should be discussed in future studies.

\section{Conflict of interest:}

None.

\section{Authors' contributions}

Xiaobin Gu and Wenhui Zhu designed the study. Haiyi He and Zili Wang participated in data collection. Shaolong Ding performed statistical analyses. Gaosheng Guo drafted the manuscript. All authors critically revised the manuscript. All authors read and approved the final manuscript.

\section{RESUMO:}

OBJETIVO: Investigar a eficácia e segurança da discectomia endoscópica percutânea transforaminal (DEPT) no tratamento da estenose lombar (EL) combinada à osteoporose.

MÉTODOS: Oitenta pacientes com EL combinada à osteoporose foram divididos entre um grupo de controle e um grupo de DEPT, que receberam tratamento convencional com fusão intersomática lombar transforaminal e DEPT, respectivamente. As indicações cirúrgicas, a escala analógica visual (VAS) da incisão e de dor lombar e nas pernas, os escores lombares de acordo com a Associação Ortopédica Japonesa (JOA) e o Oswestry Disability Index (ODI), a densidade mineral óssea (DMO) e possíveis reações adversas foram observados.

RESULTADOS: Em comparação com o grupo de controle, no grupo de DEPT o tempo de operação, a perda de sangue e duração de internação, os escores VAS da incisão no pós-operatório após 12, 24 e 48 h, o VAS para dor lombar e nas pernas e os escores ODI lombares após 6 meses de pós-operatório foram significativamente menores $(P<0,01)$; já o escore JOA lombar após 6 meses de pós-operatório foi significativamente maior $(P<0,05)$. Não houve diferença significativa na densidade mineral óssea entre os dois grupos $(P>0,05)$. Em comparação com o grupo de controle, o grupo de DEPT teve uma taxa efetiva total significativamente maior $(P<0,05)$, e a incidência de reações adversas foi significativamente menor $(P<0,05)$.

CONCLUSÕES: A discectomia endoscópica percutânea transforaminal é segura e eficaz no tratamento de EL combinada à osteoporose. PALAVRAS-CHAVE: Discectomia, percutânea. Estenose espinhal. Osteoporose. Eficácia. Segurança.

\section{REFERENCES}

1. Kreiner DS, Shaffer WO, Baisden JL, Gilbert TJ, Summers JT, Toton JF, et al; North American Spine Society. An evidence-based clinical guideline for the diagnosis and treatment of degenerative lumbar spinal stenosis (update). Spine J. 2013;13(7):734-43.

2. Grover A, LeBoff MS. Osteoporosis/vertebral compression fractures. J Korean Soc Spine Surg. 2000;8(3):242-7.

3. Kanchiku T, Imajo Y, Suzuki H, Yoshida Y, Taguchi T, Tominaga T, et al. Comparisons on efficacy of elcatonin and limaprost alfadex in patients with lumbar spinal stenosis and concurrent osteoporosis: a preliminary study using a crossover design. Asian Spine J. 2014;8(4):469-75.

4. Iwamoto J, Sato Y, Takeda T, Matsumoto H. Effectiveness of exercise in the treatment of lumbar spinal stenosis, knee osteoarthritis, and osteoporosis. Aging Clin Exp Res. 2010;22(2):116-22.
5. Gadjradj PS, van Tulder MW, Dirven CM, Peul WC, Harhangi BS. Clinical outcomes after percutaneous transforaminal endoscopic discectomy for lumbar disc herniation: a prospective case series. Neurosurg Focus. 2016;40(2):E3.

6. Gadjradj PS, Harhangi BS. Percutaneous transforaminal endoscopic discectomy for lumbar disk herniation. Clin Spine Surg. 2016;29(9):368-71.

7. Fan G, Guan X, Sun Q, Hu A, Zhu Y, Gu G, et al. Puncture reduction in percutaneous transforaminal endoscopic discectomy with HE's Lumbar LOcation (HELLO) System: a cadaver study. PLoS One. 2015;10(12):e0144939.

8. Jang JS, An SH, Lee SH. Transforaminal percutaneous endoscopic discectomy in the treatment of foraminal and extraforaminal lumbar disc herniations. | Spinal Disord Tech. 2006;19(5):338-43.

9. Klammer G, Maquieira G), Spahn S, Vigfusson V, Zanetti M, Espinosa N. 
Natural history of nonoperatively treated osteochondral lesions of the talus. Foot Ankle Int. 2015;36(1):24-31.

10. Hashimoto H, Komagata M, Nakai O, Morishita M, Tokuhashi Y, Sano S, et al. Discriminative validity and responsiveness of the Oswestry Disability Index among Japanese outpatients with lumbar conditions. Eur Spine ]. 2006;15(11):1645-50

11. Zheng CF, Liu YC, Hu YC, Xia Q, Miao J, Zhang JD, et al. Correlations of Japanese Orthopaedic Association scoring systems with gait parameters in patients with degenerative spinal diseases. Orthop Surg. 2016;8(4):447-

12. Sinkemani A, Hong X, Gao ZX, Zhuang SY, jiang ZL, Zhang SD, et al. Outcomes of microendoscopic discectomy and percutaneous transforaminal endoscopic discectomy for the treatment of lumbar disc herniation: a comparative retrospective study. Asian Spine J. 2015;9(6):833-40.

13. Sairyo K, Egawa H, Matsuura T, Takahashi M, Higashino K, Sakai T, et al.
State of the art: transforaminal approach for percutaneous endoscopic lumbar discectomy under local anesthesia. J Med Invest. 2014;61(3-4):21725.

14. Wu W, Liang J, Chen Y, Chen A, Wu B, Yang Z. Microstructural changes in compressed nerve roots treated by percutaneous transforaminal endoscopic discectomy in patients with lumbar disc herniation. Medicine (Baltimore). 2016;95(40):e5106.

15. Ahn Y. Percutaneous endoscopic decompression for lumbar spinal stenosis. Expert Rev Med Devices. 2014;11(6):605-16.

16. Wu B, Zhang S, Lian Q, Yan H, Lin X, Zhan G. Lumbar scoliosis combined lumbar spinal stenosis and herniation diagnosed patient was treated with "U" route transforaminal percutaneous endoscopic lumbar discectomy. Case Rep Orthop. 2017;2017:7439016.

17. Cleland JG, Witte K, Steel S. Calcium supplements in people with osteoporosis. BM|. 2010;341:c3856. 